\title{
Involvement of glycine binding site of NMDA receptor in the prefrontal cortex of SHRSP/Ezo as an AD/HD animal model
}

\author{
Hiroki Shikanai ${ }^{1}$, Nobuhiro Oshima ${ }^{2}$, Hidekazu Kawashima ${ }^{2}$, Shin-Ichi Kimura ${ }^{1}$, Sachiko Hiraide ${ }^{1}$, \\ Kenji lizuka ${ }^{1}$, Takeshi Izumi ${ }^{1}$ \\ ${ }^{I}$ Department of Pharmacology, Health Science University of Hokkaido, Japan, ${ }^{2}$ Department of Biophysical Sciences, \\ Health Science University of Hokkaido, Japan
}

Background: Attention deficit/ hyperactivity disorder (AD/HD) is a mild developmental disorder. We previously reported that stroke-prone spontaneously hypertensive rat/Ezo (SHRSP/Ezo) had high validity as an AD/HD animal model, because of its behavioral phenotype. It was reported that $\mathrm{AD} / \mathrm{HD}$ had the front-cortical dysfunction, hypofrontality. Based on these reports, we hypothesized that SHRSP/Ezo as $\mathrm{AD} / \mathrm{HD}$ animal model has some fronto-cortical incompetence. Thus, we evaluated the excitatory synaptic function in the PFC of SHRSP/Ezo using electrophysiological and pharmacological methods.

Methods: At 6 weeks old, SHRSP/Ezo and its genetic control, WKY/Ezo were anesthetized and immediately decapitated. Using acute coronal brain slice, we recorded excitatory postsynaptic potential (EPSP) in layer V pyramidal neurons in the PFC by whole-cell patch clamp recording method to assess synaptic plasticity in the form of long-term potentiation (LTP). We also performed N-methyl-D-aspartic acid (NMDA) receptor binding assay in the PFC and hippocampus with radio-labeled NMDA receptor antagonist $\left[{ }^{3} \mathrm{H}\right] \mathrm{MK}-801$ using homogenized membrane tissue.

Results: Theta burst stimulation induced long-lasting EPSP enhancement, LTP, in the PFC of WKY/Ezo, whereas failed to induce LTP, in that of SHRSP/Ezo. The $K_{\mathrm{d}}$ value of $\left[{ }^{3} \mathrm{H}\right] \mathrm{MK}-801$ binding for NMDA receptors in the PFC of SHRSP/Ezo was higher than in the WKY/Ezo. Neither the $B_{\max }$ nor $K_{\mathrm{d}}$ of $\left[{ }^{3} \mathrm{H}\right] \mathrm{MK}-801$ binding in the SHRSP/Ezo hippocampus were significantly different to WKY/Ezo. When we added $100 \mu \mathrm{M}$ glycine into the incubate solution to assess the function of glycine binding site of NMDA receptor in each rat, there was no significant difference of $K_{\mathrm{d}}$ value in the PFC of SHRSP/Ezo.

Conclusion: These results suggest that the AD/HD animal model SHRSP/Ezo has NMDA receptor dysfunction in the PFC, but not the hippocampus. Moreover, some ligands for glycine binding site in NMDA receptor can be a possibility of new drug for $\mathrm{AD} / \mathrm{HD}$ treatment. 